I. Barroso $\cdot$ J. Luan $\cdot$ M. S. Sandhu •

P. W. Franks $\cdot$ V. Crowley $\cdot$ A. J. Schafer .

S. O'Rahilly $\cdot$ N. J. Wareham

\title{
Meta-analysis of the Gly482Ser variant in PPARGC1A in type 2 diabetes and related phenotypes
}

Received: 1 August 2005 / Accepted: 4 November 2005 / Published online: 25 January 2006

C) Springer-Verlag 2006

\begin{abstract}
Aims/hypothesis: Peroxisome proliferator-activated receptor- $\gamma$ co-activator- $1 \alpha$ (PPARGC1A) is a transcriptional co-activator with a central role in energy expenditure and glucose metabolism. Several studies have suggested that the common PPARGC1A polymorphism Gly482Ser may be associated with risk of type 2 diabetes, with conflicting results. To clarify the role of Gly482Ser in type 2 diabetes and related human metabolic phenotypes we genotyped this polymorphism in a case-control study and performed a meta-analysis of relevant published data. Materials and methods: Gly 482Ser was genotyped in a type 2 diabetes case-control
\end{abstract}

Electronic Supplementary Material Supplementary material is available for this article at http://dx.doi.org/10.1007/s00125-0050130-2

I. Barroso $(\square)$

The Wellcome Trust Sanger Institute,

Metabolic Disease Group,

The Wellcome Trust Genome Campus,

Hinxton, Cambridgeshire CB10 1SA, UK

e-mail: ib1@sanger.ac.uk

Fax: +44-1223-494919

J. Luan · P. W. Franks · N. J. Wareham

MRC Epidemiology Unit,

Cambridge, UK

M. S. Sandhu

Department of Public Health \& Primary Care,

Institute of Public Health, University of Cambridge,

Cambridge, UK

P. W. Franks

Phoenix Epidemiology \& Clinical Research Branch,

NIDDK, NIH,

Phoenix, AZ USA

V. Crowley $\cdot$ S. O'Rahilly

Departments of Clinical Biochemistry and Medicine, Addenbrooke's Hospital,

Cambridge, UK

A. J. Schafer

Incyte Genomics,

Cambridge, UK study $(N=1,096)$ using MassArray technology. A literature search revealed publications that examined Gly482Ser for association with type 2 diabetes and related metabolic phenotypes. Meta-analysis of the current study and relevant published data was undertaken. Results: In the pooled meta-analysis, including data from this study and seven published reports (3,718 cases, 4,818 controls), there was evidence of between-study heterogeneity $(p<0.1)$. In the fixed-effects meta-analysis, the pooled odds ratio for risk of type 2 diabetes per Ser482 allele was 1.07 (95\% CI $1.00-1.15, p=0.044)$. Elimination of one of the studies from the meta-analysis gave a summary odds ratio of $1.11(95 \%$ CI $1.04-1.20, p=0.004)$, with no between-study heterogeneity $(p=0.475)$. For quantitative metabolic traits in normoglycaemic subjects, we also found significant between-study heterogeneity. However, no significant association was observed between Gly482Ser and BMI, fasting glucose or fasting insulin. Conclusions/ interpretation: This meta-analysis of data from the current and published studies supports a modest role for the Gly482Ser PPARGC1A variant in type 2 diabetes risk.

Keywords Association study · Meta-analysis · Polymorphism · PPARGC1A · Type 2 diabetes

Abbreviations OR: odds ratio PPAR: peroxisome proliferator-activated receptor PPARGC1A: peroxisome proliferator-activated receptor- $\gamma$ co-activator- $1 \alpha \cdot$ SNP: single-nucleotide polymorphism

\section{Introduction}

Peroxisome proliferator-activated receptor- $\gamma$ co-activator$1 \alpha$ (PPARGC1A) is a potent transcriptional co-activator of nuclear receptors, including PPAR- $\alpha$ and PPAR- $\gamma$, and controls transcription of genes involved in adaptive thermogenesis, adipogenesis and oxidative metabolism [1-3]. PPARGC1A also plays a key role in the regulation of hepatic glucose output through the control of gluconeogenesis $[4,5]$, and modulates the expression of the insulin- 
regulated glucose transporter GLUT4 through an interaction with the transcription factor MADS box transcription enhancer factor 2, polypeptide C (also known as MEF2C) [6]. Moreover, decreased PPARGC1A expression was detected in muscle of individuals with diabetes and in unaffected subjects with a family history of diabetes [7].

In view of its extensive role in metabolism, PPARGC1A has been considered a candidate gene for type 2 diabetes and other intermediary metabolic phenotypes. Specifically, a common PPARGC1A polymorphism, Gly482Ser, has been studied for association with type 2 diabetes with conflicting results [8-12]. The Gly482Ser variant has also been associated with insulin resistance [9], indices of obesity in women [13], and mean insulin secretory response and lipid oxidation [11]. More recently, a report found an association between specific PPARGC1A haplotypes and 30-min and 60-min post-load glucose levels and indices of beta cell function, and a borderline significant association with type 2 diabetes; however, no one single-nucleotide polymorphism (SNP), including Gly482Ser, was significantly associated with type 2 diabetes [14]. Haplotypes of PPARGC1A have also been found to be associated with indices of glucose tolerance in non-diabetic offspring of type 2 diabetes subjects [15]. Considering the high plausibility of PPARGC1A as a functional candidate gene for type 2 diabetes predisposition, and in light of the conflicting results from earlier studies, we evaluated the role of the Gly482Ser polymorphism in PPARGC1A in type 2 diabetes and related metabolic phenotypes.

It has been demonstrated that to attain sufficient power to establish the role of certain polymorphisms and the risk of disease many thousands of samples are often required. Frequently, these samples are not available in a single study, and meta-analysis is used to pool together the results from several different studies. In type 2 diabetes, meta-analysis has been used successfully to establish the role of risk alleles for type 2 diabetes [16-19]. Towards this end, we have combined results from genotyping the Gly482Ser variant in our current study with those from available published studies and performed a meta-analysis of the dataset.

\section{Subjects and methods}

European prospective investigation of cancer-Norfolk subjects

This is a nested case-control study within the European Prospective Investigation of Cancer (EPIC) - Norfolk prospective cohort study; both the case-control [20] and full cohort study $[20,21]$ have previously been described in detail. Briefly, the case-control study consists of 417 incident type 2 diabetes cases and two sets of 417 controls, each matched in terms of age, sex, time in study and family physician, with one set additionally matched for BMI. A case was defined by a doctor's diagnosis of type 2 diabetes, with no insulin prescribed within the first year after diagnosis, and/or $\mathrm{HbA}_{1} \mathrm{c}>7 \%$ at baseline or the follow-up health check. Controls were selected from those in the cohort who had not reported diabetes, cancer, stroke or myocardial infarction at baseline, and who had not developed diabetes by the time of selection. Potential controls with measured $\mathrm{HbA}_{1} \mathrm{c}$ levels $>6 \%$ were excluded. DNA was available for this analysis from 355 cases and 741 controls. Informed consent was granted by all participating subjects and ethical approval for the study was granted by the Cambridge Local Research Ethics Committee.
Fig. 1 Odds ratios and 95\% CIs for case-control studies and meta-analysis for an additive model. The squares represent the point estimates of the odds ratio for each study. The area of each is proportional to the percentage weight of each study in the overall meta-analysis. Horizontal lines represent the 95\% CI for each individual study. The overall $95 \% \mathrm{CI}$ for the meta-analysis is represented by the diamond. Genotype counts for subjects with type 2 diabetes $(D)$ and normoglycaemic controls $(C)$ are shown for each study as Gly/Gly / Gly/Ser/ Ser/Ser. Overall OR $=1.071$ (95\% CI 1.002-1.145), $p=0.044$. Heterogeneity test: $p=0.069$
1. Ek et al., 2001 [8] D: $186 / 200 / 68$; C: $97 / 80 / 21$

2. Ek et al.. 2001 [8] D: $76 / 97 / 28 ;$ C: $146 / 116 / 31$

3. Hara et al., 2002 [9] D: $119 / 207 / 91$; C: 178/249/110

4. Lacquemant et al., 2002 [10] D: $280 / 284 / 95$; C: $323 / 327 / 98$

5. Muller et al., 2003 [11] D: $415 / 198 / 29 ;$ C: $219 / 116 / 7$

6. Barroso et al., 2003 [12] D: $230 / 226 / 58$; C: 211/215/81

8. Oberkofler et al., 2004 [14] D: 200/228/66; C: 624/679/175

9. EPIC

D: $130 / 175 / 32$; C: $307 / 328 / 78$

Overall $95 \% \mathrm{Cl}$

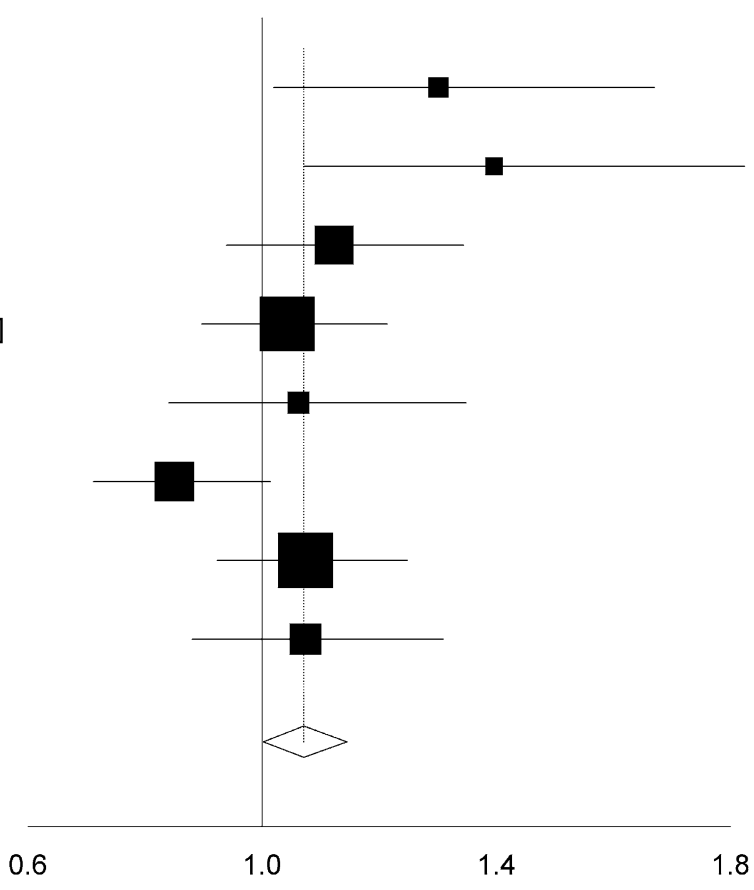


Genotyping

Samples were arrayed on 96-well plates, with three replicates and one water control per plate. Genotyping of samples was performed at the Wellcome Trust Sanger Institute, Cambridge, using an adaptation of the homogenous MassExtend protocol supplied by Sequenom for the Mass Array system (Sequenom, San Diego, CA, USA) [22]. The genotyping success rate was $95.8 \%$. There were no discordancies in replicate samples and results were in agreement with Hardy-Weinberg equilibrium.

Literature searches A literature search was performed using the search terms 'peroxisome proliferator activator receptor gamma coactivator 1', 'PPARGC1', 'PGC1', 'PGC-1', 'PGC-1alpha' and 'PGC1alpha' with the AND search terms 'polymorphism', 'SNP' and 'association' to identify all genetic association studies published for this gene. This search included articles published up to June 2005. All studies that tested the Gly482Ser polymorphism for association with type 2 diabetes were included in this study, as well as those that published genotype counts and included data on fasting glucose, fasting insulin and BMI in normoglycaemic subjects. Data from these manuscripts was collated for meta-analysis, in a few instances the authors of a paper were contacted to obtain the original genotype distribution data when this data was not included in the original article $[9,14]$.

Statistical analysis All statistical analyses were performed using the SAS statistical analysis software for Windows (version 8.02; SAS Institute, Cary, NC, USA) unless otherwise stated. For case-control studies, logistic regression was used to estimate odds ratios (OR). Simple linear regression was used to assess the association between genotype and relevant metabolic traits. In all primary

Table 1 Mean values for BMI, fasting insulin and fasting glucose in non-diabetic subjects according to Gly482Ser genotype

\begin{tabular}{|c|c|c|c|c|c|c|c|}
\hline \multirow[b]{2}{*}{ Study [Ref] } & \multirow[b]{2}{*}{ Parameter } & \multirow[b]{2}{*}{ Gly/Gly } & \multirow[b]{2}{*}{ Gly/Ser } & \multirow[b]{2}{*}{ Ser/Ser } & \multicolumn{3}{|l|}{ Linear trend test } \\
\hline & & & & & Beta $(95 \% \mathrm{CI})$ & $p$ value & \\
\hline \multirow[t]{2}{*}{ Mean (SD) } & Age (years) & $51(14)$ & $53(13)$ & $50(14)$ & & & \\
\hline & BMI $\left(\mathrm{kg} / \mathrm{m}^{2}\right)$ & $25.4(4)$ & $25.3(3.7)$ & $24.2(3.6)$ & $-0.6(-4.27$ to 3.07$)$ & 0.285 & \\
\hline $2[8]$ & Sex (men/women) & $68 / 78$ & $49 / 67$ & $17 / 14$ & & & \\
\hline \multirow[t]{4}{*}{ Mean (SD) } & Age (years) & $61(0.5)$ & $61(0.4)$ & $60(0.4)$ & & & \\
\hline & BMI $\left(\mathrm{kg} / \mathrm{m}^{2}\right)$ & $25.9(3.2)$ & $26.5(4.2)$ & $26(3.6)$ & $0.05(-3.99$ to 4.09$)$ & 0.901 & \\
\hline & $\mathrm{FPI}(\mathrm{pmol} / \mathrm{l})^{\mathrm{a}}$ & $39.1(19.2)$ & $39.9(25.1)$ & $37.6(16)$ & $-0.75(-12.1$ to 10.6$)$ & 0.556 & \\
\hline & FPG $(\mathrm{mmol} / \mathrm{l})$ & $5.2(0.5)$ & $5.1(0.5)$ & $5.1(0.5)$ & $-0.05(-0.42$ to 0.32$)$ & 0.333 & \\
\hline Mean (SEM) & FPG (mmol/l) & $5.21(0.04)$ & $5.16(0.04)$ & $5.23(0.05)$ & $0.01(-0.43$ to 0.45$)$ & 0.821 & \\
\hline $7[12]$ & Sex (men/women) & $127 / 202$ & $184 / 228$ & $43 / 62$ & & & \\
\hline \multirow[t]{4}{*}{ Mean (SEM) } & Age (years) & $55.1(0.593)$ & $54.2(0.526)$ & $55.3(1.04)$ & & & \\
\hline & BMI $\left(\mathrm{kg} / \mathrm{m}^{2}\right)$ & $26.7(0.239)$ & $26.3(0.212)$ & $26.6(0.42)$ & $-0.05(-2.62$ to 2.52$)$ & 0.846 & \\
\hline & $\mathrm{FPI}(\mathrm{pmol} / \mathrm{l})^{\mathrm{a}}$ & $41.4(2.065)$ & $39.8(1.861)$ & $38.1(3.667)$ & $-1.65(-2.02$ to -1.28$)$ & $0.011^{\mathrm{c}}$ & \\
\hline & FPG $(\mathrm{mmol} / \mathrm{l})$ & $4.92(0.04)$ & $4.87(0.036)$ & $4.85(0.071)$ & $-0.035(-0.15$ to 0.08$)$ & 0.154 & \\
\hline $8[14]^{\mathrm{b}}$ & Sex (men/women) & $382 / 242$ & $423 / 256$ & $113 / 62$ & & & \\
\hline \multirow[t]{3}{*}{ Mean (SD) } & Age (years) & 51.7 & 51.5 & 51.5 & & & \\
\hline & BMI $\left(\mathrm{kg} / \mathrm{m}^{2}\right)$ & $26.6(4.07)$ & $26.4(3.94)$ & $26.4(3.88)$ & $-0.14(-0.99$ to 0.72$)$ & 0.29 & \\
\hline & FPI (pmol/l) & $48.6(29.5)$ & $49.0(31.4)$ & $45.6(25.0)$ & $-1.51(-15.1$ to 12.1$)$ & 0.392 & \\
\hline
\end{tabular}

The calculated $Q$ statistic for test of heterogeneity for fasting insulin levels (4 $d f)$ was 21.9

FPI Fasting plasma insulin; FPG fasting plasma glucose

${ }^{\text {a }}$ Logarithmically transformed values

${ }^{\mathrm{b}}$ Pooled means and standard deviations from the original data according to sex

${ }^{\mathrm{c}} p$ values $<0.05$ 
analyses, we assumed a linear relation between the number of Ser alleles and the outcome or trait of interest. For the fixed-effects meta-analysis, pooled odds ratios for casecontrol studies and regression coefficients for quantitative traits studies were calculated using a variance-based method, weighting individual study results by the inverse of their variance. Results were considered significant at $p<0.05$. Heterogeneity among studies was assessed using the $Q$ statistic. Results were considered heterogeneous when homogeneity was unlikely $(p<0.10)$. To determine the stability of the summary risk estimates, we performed a sensitivity analysis based on the successive elimination of each study.

\section{Results}

The details of published studies included in the current meta-analysis are summarised in Table 1 of the Electronic Supplementary Material. Genotype counts in cases and controls in the current study are represented in Fig. 1 (study 9). In the pooled dataset, which included all published data, the genotype distribution of the Gly482Ser polymorphism was similar in subjects with type 2 diabetes and controls $\left(\chi_{2 d f}^{2}=4.67, p=0.097\right)$. The difference in the frequency of the Ser482 allele between cases and controls in the pooled dataset did reach nominal significance $\left(\chi_{1}^{2} d f=4.17\right.$, $p=0.041)$. In the fixed-effects meta-analysis, which included eight studies, the pooled odds ratio for risk of type 2 diabetes per Ser allele was 1.07 (95\% CI 1.00-1.15, $p=0.044)$. As noted in Fig. 1, a test for heterogeneity among studies was statistically significant $(Q=13.2,7 d f$, $p=0.069$ ). In a sensitivity analysis, the summary odds ratio for risk of type 2 diabetes varied between 1.05 and 1.11 in an additive model (data not shown). In these analyses, exclusion of study 6 gave a summary OR of $1.11(95 \%$ CI $1.04-1.20, p=0.004)$, and there was no between-study heterogeneity $(Q=5.6,6 d f, p=0.475)$.

We also tested whether the Gly482Ser genotype influences relevant clinical phenotypes, such as BMI, fasting glucose and fasting insulin, in a total of 2,910 men and 1,576 women without type 2 diabetes. For each study population, mean values for BMI, fasting insulin and glucose according to Gly482Ser genotype in participants without diabetes are shown in Table 1 . For these quantitative traits, there was no consistent association between metabolic factors and genotype in a pooled analysis. However, we found significant between-study heterogeneity for two metabolic traits (fasting insulin: $Q=21.9,4 d f$, $p=0.0002$; and BMI: $Q=8.0,4 d f, p=0.092$ ). In a sensitivity analysis, we found that exclusion of study 3 lead to the elimination of the between-study heterogeneity; however, no major effects were observed in these quantitative traits.

\section{Discussion}

The current meta-analysis, which includes data on 8,536 participants, indicates that the PPARGC1A Ser482 allele may increase the risk of type 2 diabetes. It is possible, however, that publication bias may explain the observed association between this genetic variant and risk of type 2 diabetes. We could not detect any statistically significant association of the Gly482Ser polymorphism with BMI, fasting insulin or fasting glucose levels in a meta-analysis comprised of 4,486 normoglycaemic study participants.

Over the last 6 years the role of PPARGC1A in several aspects of energy expenditure, metabolism and glucose homeostasis has been elucidated. This has led to several studies assessing the association among polymorphisms, risk of type 2 diabetes and related metabolic traits. The only common non-synonymous change, the Gly482Ser polymorphism, has generated conflicting results. In this study we aimed to address this issue by performing a metaanalysis of the effect of the Gly482Ser polymorphism on type 2 diabetes, BMI, fasting insulin and fasting glucose levels.

In this meta-analysis, which included data from eight studies comprising 8,536 participants, there was evidence for a small linear effect of the Gly482Ser in type 2 diabetes risk $(\mathrm{OR}=1.07, p=0.044)$, despite the presence of marginal between-study heterogeneity $(p=0.069)$. The summary odds ratio increased to $1.1(p=0.004)$ and there was no between-study heterogeneity after elimination of one of the studies. We also found significant between-study heterogeneity in the quantitative trait analyses. However, in this analysis we found no statistically significant association between the Gly482Ser polymorphism and the three traits analysed (BMI, fasting insulin and fasting glucose). In each instance the heterogeneity observed in the association with type 2 diabetes or with the quantitative traits could be explained by a different constituent population of the metaanalysis. This heterogeneity could result from differences in other genetic and environmental factors between study populations, particularly in relation to selection and inclusion of participants and their demographic, metabolic and clinical characteristics. Other possible explanations for the observed between-study heterogeneity include genotype errors, publication bias, lack of statistical power to detect true effects and simple random variation around the true estimate of risk.

The variable effect of the Gly482Ser polymorphism on type 2 diabetes risk and related quantitative traits between populations could also be explained by the presence of other functional variants in linkage disequilibrium (LD) with Gly482Ser. The amount of LD between these putative variants and Gly482Ser could vary between the different populations tested, hence accounting for the observed differences in the effect of Gly482Ser in diabetes association and in mean values for phenotypic traits between populations. Other possible explanations for the heterogeneity observed are gene-gene and geneenvironment interactions such that the effect of the Gly482Ser polymorphism is different in different populations. Indeed, evidence for gene-environment interaction at this gene has been demonstrated [23-25]. Geneenvironment interactions may lower the power to detect true associations when performing cross-sectional studies, 
including meta-analysis of cross-sectional studies, such as in this study.

In conclusion, our results, based on 8,536 study participants, suggest that the PPARGC1A Gly482Ser polymorphism is associated with risk of type 2 diabetes. However, larger scale studies are required to reliably confirm any association between this variant and the risk of type 2 diabetes.

Acknowledgements This work was supported in part by The Wellcome Trust (I. Barroso, S. O'Rahilly, N. J. Wareham), and the Medical Research Council (N. J. Wareham, P. W. Franks, M. S. Sandhu). We also thank S. Bumpstead for technical assistance. The authors declare no conflicts of interest.

\section{References}

1. Puigserver P, Wu Z, Park CW et al (1998) A cold-inducible coactivator of nuclear receptors linked to adaptive thermogenesis. Cell 92:829-839

2. Wu Z, Puigserver P, Andersson U et al (1999) Mechanisms controlling mitochondrial biogenesis and respiration through the thermogenic coactivator PGC-1. Cell 98:115-124

3. Puigserver P, Spiegelman BM (2003) Peroxisome proliferatoractivated receptor-gamma coactivator 1 alpha (PGC-1 alpha): transcriptional coactivator and metabolic regulator. Endocr Rev 24:78-90

4. Herzig S, Long F, Jhala US et al (2001) CREB regulates hepatic gluconeogenesis through the coactivator PGC-1. Nature 413:179-183

5. Yoon JC, Puigserver P, Chen G et al (2001) Control of hepatic gluconeogenesis through the transcriptional coactivator PGC-1. Nature 413:131-138

6. Michael LF, Wu Z, Cheatham RB et al (2001) Restoration of insulin-sensitive glucose transporter (GLUT4) gene expression in muscle cells by the transcriptional coactivator PGC-1. Proc Natl Acad Sci USA 98:3820-3825

7. Patti ME, Butte AJ, Crunkhorn S et al (2003) Coordinated reduction of genes of oxidative metabolism in humans with insulin resistance and diabetes: potential role of PGC1 and NRF1. Proc Natl Acad Sci USA 100:8466-8471

8. Ek J, Andersen G, Urhammer SA et al (2001) Mutation analysis of peroxisome proliferator-activated receptor-gamma coactivator-1 (PGC-1) and relationships of identified amino acid polymorphisms to Type II diabetes mellitus. Diabetologia 44:2220-2226

9. Hara K, Tobe K, Okada T et al (2002) A genetic variation in the PGC-1 gene could confer insulin resistance and susceptibility to Type II diabetes. Diabetologia 45:740-743

10. Lacquemant C, Chikri M, Boutin P et al (2002) No association between the G482S polymorphism of the proliferator-activated receptor-gamma coactivator-1 (PGC-1) gene and Type II diabetes in French Caucasians. Diabetologia 45:602-3; author reply 604
11. Muller YL, Bogardus C, Pedersen O et al (2003) A Gly482Ser missense mutation in the peroxisome proliferator-activated receptor gamma coactivator-1 is associated with altered lipid oxidation and early insulin secretion in Pima Indians. Diabetes. 52:895-898

12. Barroso I, Luan J, Middelberg RP et al (2003) Candidate gene association study in type 2 diabetes indicates a role for genes involved in beta-cell function as well as insulin action. PLoS Biol 1:E20

13. Esterbauer H, Oberkofler H, Linnemayr V et al (2002) Peroxisome proliferator-activated receptor-gamma coactivator1 gene locus: associations with obesity indices in middle-aged women. Diabetes 51:1281-1286

14. Oberkofler H, Linnemayr V, Weitgasser R et al (2004) Complex haplotypes of the PGC-1alpha gene are associated with carbohydrate metabolism and type 2 diabetes. Diabetes 53:1385-1393

15. Pihlajamäki J, Kinnunen M, Ruotsalainen E et al (2005) Haplotypes of PPARGC1A are associated with glucose tolerance, body mass index and insulin sensitivity in offspring of patients with type 2 diabetes. Diabetologia 48:1331-1334

16. Altshuler D, Hirschhorn JN, Klannemark M et al (2000) The common PPARgamma Pro12Ala polymorphism is associated with decreased risk of type 2 diabetes. Nat Genet 26:76-80

17. Hani EH, Boutin P, Durand E et al (1998) Missense mutations in the pancreatic islet beta cell inwardly rectifying $\mathrm{K}+$ channel gene (KIR6.2/BIR): a meta-analysis suggests a role in the polygenic basis of Type II diabetes mellitus in Caucasians. Diabetologia 41:1511-1515

18. Gloyn AL, Weedon MN, Owen KR et al (2003) Large-scale association studies of variants in genes encoding the pancreatic beta-cell KATP channel subunits Kir6.2 (KCNJ11) and SUR1 (ABCC8) confirm that the KCNJ11 E23K variant is associated with type 2 diabetes. Diabetes 52:568-572

19. Nielsen EM, Hansen L, Carstensen B et al (2003) The E23K variant of Kir6.2 associates with impaired post-OGTT serum insulin response and increased risk of type 2 diabetes. Diabetes 52:573-577

20. Harding A-H (2001) The role of dietary fat in the aetiology of type 2 diabetes mellitus. PhD thesis, Department of Public Health and Primary Care, University of Cambridge

21. Day N, Oakes S, Luben R et al (1999) EPIC-Norfolk: study design and characteristics of the cohort. European prospective investigation of cancer. Br J Cancer 80(Suppl 1):95-103

22. Whittaker P, Bumpstead S, Downes K, Ghori J, Deloukas P (2006) SNP analysis by MALDI-TOF mass spectrometry. In: Celis J (ed) Cell biology: a laboratory handbook. Academic, San Diego

23. Andrulionyte L, Zacharova J, Chiasson JL et al (2004) Common polymorphisms of the PPAR-gamma2 (Pro12Ala) and PGC-1alpha (Gly482Ser) genes are associated with the conversion from impaired glucose tolerance to type 2 diabetes in the STOP-NIDDM trial. Diabetologia 47:2176-2184

24. Franks PW, Barroso I, Luan J et al (2003) PGC-1alpha genotype modifies the association of volitional energy expenditure with VO2max. Med Sci Sports Exerc 35: 1998-2004

25. Lucia A, Gomez-Gallego F, Barroso I et al (2005) PPARGC1 genotype (Gly482Ser) predicts exceptional endurance capacity in European males. J Appl Physiol 99:344-348 